\title{
Estrategia metodológica para abordar procesos etnoeducativos orientada a contribuir en la transformación de situaciones ambientales en comunidades negras del Valle del Cauca, Colombia
}

\section{Methodological strategy to address ethniceducation oriented processes contribute in the transformation of environmental situations. Case: black communities Andean valleys of Valle del Cauca, Colombia}

\author{
Carlos Ariel Rentería Jiménez*
}

\section{Resumen}

Se presenta una estrategia metodológica para abordar procesos educativos orientada a contribuir en la transformación de situaciones ambientales, específicamente en territorios de Consejos Comunitarios de los Valles Interandinos, cuenca Cauca, en el departamento del Valle del Cauca, Colombia, con el propósito de proponer una herramienta práctica y de uso sencillo para ayudar a las organizaciones públicas o privadas a entender la necesidad de conocer y respetar las complejidades culturales, sociales, económicas y políticas relacionadas con la realización de proyectos y demás actividades en territorios colectivos de comunidades negras. En función de este objetivo, el trabajo se estructura de la siguiente manera: un marcoteórico relacionado con las claves de la investigación; la propuesta de estrategia metodológica para abordar procesos educativos orientada a contribuir en la transformación de situaciones ambientales; la validación de la propuesta metodológica en las comunidades negras de los Consejos Comunitarios de los Valles Interandinos; $y$, por último, se presentan algunas consideraciones finales a manera de conclusiones.

Palabras clave: Bienestar colectivo, Comunidades negras, Consejos comunitarios, Diálogo intercultural.

\begin{abstract}
This article presents a methodological strategy is presented to address educational processes oriented to contribute to the transformation of environmental situations, specifically in areas of Community Councils located in the valleys, basin Cauca in the department of Valle del Cauca, in order to propose a practical and easy to use tool to assist public and private organi-
\end{abstract}

* Investigador de proyectos especiales, Instituto de Investigaciones Ambientales del Pacífico (IIAP). Magister en Docencia de la Química, Quibdó, Chocó, Colombia. e-mail: carielrenteria@hotmail.com 
zations understand the need to know and respect the cultural, social, economic and political complexities related to the implementation of projects and other activities in collective territories of black communities. Based on this objective, the work is structured as follows: a theoretical framework related to key research; the proposal to address methodological strategy aimed at contributing to the transformation of educational processes environmental situations; the validation of the methodology in the black communities of the Community Councils of the Valleys; and finally, some final considerations by way of conclusions.

Keywords: Black communities, Community councils, Collective welfare, Intercultural dialogue.

\section{Introducción}

El desarrollo del presente trabajo se dio en el marco de la alianza estratégica de cooperación horizontal entre el Instituto de Investigaciones Ambientales del Pacífico (IIAP) y la Corporación Autónoma Regional del Valle del Cauca (CVC), que entre otras cosas se propuso la implementación de procesos etnoeducativos en territorios de comunidades negras del Valle del Cauca, conducentes a avanzar en la implementación de acciones tendientes a mejorar su entorno natural. Para esto es necesario desarrollar estrategias que permitan a los entes gubernamentales, organizaciones públicas o privadas y demás actores sociales y comunitarios contar con una herramienta de interacción comunicativa que permita mejorar los niveles de acercamiento y relacionamiento con las comunidades afrodescendientes organizadas en Consejos Comunitarios en el Valle del Cauca, a fin de generar una relación de trabajo fundamentada en la confianza, con la finalidad de potenciar sinergias para su bienestar.

Cualquier ejercicio de relacionamiento comunitario implica definir en un primer momento qué se entiende por cultura. No obstante, por la complejidad del término y su amplio abanico de interpretaciones, conviene dejar claro que el sentido que aquí se usará es el propuesto por la Organización de las Naciones Unidas para la Educación, la Ciencia y la Cultura(UNESCO) que adoptó una definición de cultura desarrollada en la parte introductoria de la Declaración Universal de la UNESCO (2001) sobre la Diversidad Cultural:

(...) La cultura debe ser considerada como el conjunto de los rasgos distintivos espirituales y materiales, intelectuales y afectivos que caracterizan a una sociedad o a un grupo social y que abarca, además de las artes y las letras, los modos de vida, las maneras de vivir juntos, los sistemas de valores, las tradiciones y las creencias" (UNESCO, 2001).

Las culturas no son estáticas, están en constante cambio; ello ha sido reconocido en la Conferencia General de la UNESCO (2005):

Considerando que la cultura adquiere formas diversas a través del tiempo y el espacio y que esta diversidad se manifiesta en la originalidad y la pluralidad de las identidades y en las expresiones culturales de los pueblos y sociedades que forman la humanidad (UNESCO, 2005).

Las culturas no se encuentran aisladas unas de otras, porque usualmente se relacionan e influyen. Es así que la Convención sobre la Protección y Promoción de la Diversidad de las Expresiones Culturales (2005), define a la interculturalidad como "la presencia e interacción equitativa de las diversas culturas y la posibilidad de generar expresiones culturales compartidas, adquiridas por medio del diálogo y de una actitud de respeto mutuo" (p.5).

En ese mismo sentido, la interculturalidad como principio fundamental que orienta cualquier ejercicio de relacionamiento o concertación con 


\section{Bioetnia Volumen 12, 2015}

consejos comunitarios de comunidades negras, se define como un proceso que abarca el intercambio abierto y respetuoso de opiniones entre personas y grupos con diferentes tradiciones y orígenes étnicos, culturales, religiosos y lingüísticos, en un espíritu de entendimiento y respeto mutuos.

Entonces, UNESCO reconoce que uno de los principales retos de la interculturalidad es lograr que cada interlocutor de un diálogo intercultural, sea un representante del Gobierno, de Consejos Comunitarios, pueblos indígenas, de la sociedad civil y en general cualquier ciudadano, pueda relacionarse adecuadamente, lo cual implica contar con varias capacidades de comunicación.

El diálogo intercultural depende en gran medida de las competencias interculturales, que se definen como el conjunto de capacidades necesarias para relacionarse adecuadamente con los que son diferentes de nosotros. Esas capacidades son de carácter fundamentalmente comunicativo, pero también comprenden la reconfiguración de nuestros puntos de vista y nuestras concepciones del mundo, porque no son tanto las culturas, sino las personas (individuos y grupos, con su complejidad y sus múltiples lealtades) las que participan en los procesos de diálogo.

El éxito del diálogo intercultural no depende tanto del conocimiento de los otros como de la capacidad básica de escuchar, la flexibilidad cognitiva, la empatía, la humildad y la hospitalidad (...) No debería verse el diálogo como una pérdida de lo propio, sino como algo que depende del conocimiento de uno mismo y la capacidad de pasar de un marco de referencia a otro. Requiere el fortalecimiento de la autonomía de todos los participantes mediante el fomento de capacidades y proyectos que permitan la interacción, sin perjuicio de la identidad personal o colectiva (UNESCO, 2009).

De lo expuesto, el principio de interculturalidad se desarrolla mediante un proceso permanente de interrelación que parte de la interacción entre las culturas a través del respeto y reconocimiento
(SERVINDI, 2005):

- Diferencias y convergencias entre las personas y grupos

- Identidades individuales y colectivas de cada cultura como aporte complementario de otras culturas

- Derechos humanos

- Valores e intereses compartidos

- Normas de convivencia legitimadas y aceptadas

- Instituciones

- Equidad de género

- Medio ambiente

Asimismo, se debe entender a la interculturalidad como un proceso permanente de relación, comunicación y aprendizaje entre personas, grupos, conocimientos, valores y tradiciones distintas, orientadas a generar, construir y propiciar el respeto mutuo y el desarrollo pleno de las capacidades de los individuos por encima de las diferencias sociales y culturales.

Por otro lado, cabe mencionar que la interculturalidad, es un principio reconocido en el marco normativo colombiano; ello se evidencia en un primer momento en la Constitución Política de Colombia de 1991 que señala, en su Art. 7 "El Estado reconoce y protege la diversidad étnica y cultural de la Nación Colombiana". En este sentido, la presente memoria comunicativa tiene por objeto mostrar la construcción de un instrumento que de manera participativa gestione y oriente adecuadamente las relaciones entre los Consejos Comunitarios de Comunidades Negras del Valle del Cauca y la CVC, entendidos como socios estratégicos para el desarrollo de procesos educativos orientados a contribuir en la transformación de las situaciones ambientales que alteran la base natural de sus territorios, el cual se encuentra imbricado en la interculturalidad y el diálogo intercultural que como elementos fundamentales en la interacción entre las culturas se enmarcan en el respeto y reconocimiento. 


\section{Estrategia metodológica}

Para la construcción de la estrategia metodológica para abordar procesos educativos orientada a contribuir en la transformación de situaciones ambientales en Consejos Comunitarios de Comunidades Negras del Valle del Cauca, se llevaron a cabo tres fases concatenadas en las cuales se utilizó un abordaje cualitativo de investigación-acción participativa, que mediante la realización de seis talleres que involucró a los miembros de las Juntas Directivas de los Consejos Comunitarios y que fueron guiados por investigadores del IIAP.

La primera fase comprendió la selección de participantes, referida especialmente a los consejos comunitarios con los cuales se desarrolló el ejercicio de construcción, socialización y validación de la estrategia.

La segunda fase tiene que ver en primer lugar, con la identificación de los principios que podrían consolidar un proceso de relacionamiento con consejos comunitarios dirigidos a establecer un clima de confianza y respeto mutuo entre las partes y que guían la aplicación de la estrategia; en segundo lugar se fundamenta en el hecho que, para iniciar una adecuada y armoniosa relación con los hombres y mujeres de las comunidades negras, es necesario comprender los elementos que los distinguen de manera particular, considerando sus propias percepciones y conceptos acerca del mundo, de la naturaleza y formas de vida que constituyen su cultura. Por ello, es necesario conocer los elementos relevantes que deben ser considerados de la cultura afro en relación con la gestión de los recursos naturales. En tercer lugar, y desprendiéndose del hecho que los pueblos afrodescedientes tienen un arraigo espiritual con sus territorios, en los que pueden desarrollar sus prácticas tradicionales y costumbres y mantienen su cultura, se identificaran los aspectos de sensibilidad cultural que los identifican y caracterizan. Finalmente, se definen los principios y pautas claves de relacionamiento, al igual que los meca- nismos de negociación y suscripción de acuerdos con los consejos comunitarios.

La cuarta fase, se basó en las reflexiones de los participantes, los ajustes que se realizaron en el desarrollo de las fases anteriores y por último la percepción que tenían todos los actores frente a los resultados obtenidos, las sugerencias y recomendaciones que permitieron socializar y validar la estrategia metodológica para abordar procesos educativos orientada a contribuir en la transformación de situaciones ambientales en Consejos Comunitarios de Comunidades Negras del Valle del Cauca.

\section{Resultados y discusión}

Selección de participantes. En el marco de la alianza estratégica de cooperación horizontal entre el IIAP y la CVC, entre otras cosas se propuso la implementación de procesos etnoeducativos en comunidades negras del Valle del Cauca, conducentes a avanzar en la ejecución de acciones por parte de los Consejos Comunitarios de Comunidades Negras de los Valles Interandinos (cuenca Cauca), para mejorar su entorno natural: San Antonio y El Castillo; Afrozar; Paso de la Bolsa; Bocas del Palo; Tarragona Alta; Cañas Abajo; Bolo Artonal; y La Granja (Tabla 1). Para esto es necesario desarrollar estrategias que permitan a la CVC mejorar los niveles de acercamiento y relacionamiento con estas comunidades afrodescendientes a fin de generar una relación de trabajo fundamentada en la confianza, con la finalidad de potenciar sinergias para su bienestar.

\section{Principios sugeridos para el relacionamiento} con las comunidades negras. Con el fin de lograr consolidar un proceso de relacionamiento con los consejos comunitarios y en el entendido que cualquier relación se sustenta en unos principios de relacionamiento dirigidos a establecer un clima de confianza y respeto mutuo entre las partes, se lograron identificar de manera participativa los siguientes principios de relacionamiento con 


\section{Bioetnia Volumen 12, 2015}

Tabla 1. Consejos comunitarios con los cuales se construyó y validó la estrategia

\begin{tabular}{|c|c|c|c|}
\hline Consejo comunitario & Municipio & Localización & Coordenadas \\
\hline Bocas del Palo & Jamundí & $\begin{array}{l}\text { Corregimientos de Bocas del Palo y } \\
\text { las veredas de Colindares, La Isla y } \\
\text { Santa Bárbara }\end{array}$ & $\begin{array}{l}03^{\circ} 12^{\prime} 02^{\prime \prime} \text { Latitud Norte y } 76^{\circ} 28^{\prime} 18^{\prime \prime} \\
\text { Longitud Oeste }\end{array}$ \\
\hline San Antonio y El Castillo & El Cerrito & $\begin{array}{l}\text { Corregimiento El Castillo, Vereda la } \\
\text { Reina, Cuenca media del río Saba- } \\
\text { letas }\end{array}$ & $\begin{array}{l}\text { Longitud } 74^{\circ} 04^{\prime \prime} 51.3012 " \text { y Latitud } \\
04^{\circ} 3556.5692^{\prime \prime}\end{array}$ \\
\hline Paso de la Bolsa & Jamundí & Corregimiento de Paso de la Bolsa & $\begin{array}{l}\text { Latitud de } 3^{\circ} 10^{\prime} \text { Norte, Longitud de } \\
76^{\circ} 29^{\prime} \text { oeste }\end{array}$ \\
\hline La Granja & Pradera & Corregimiento de La Granja & $\begin{array}{l}04^{\circ} 35^{\prime} 46^{\prime \prime} \text { Latitud Norte y } 77^{\circ} 04^{\prime} 39^{\prime \prime} \\
\text { Longitud Oeste }\end{array}$ \\
\hline Afrozar & Zarzal & $\begin{array}{l}\text { Corregimiento de La Paila y las ve- } \\
\text { redas de La Miel, El Negro, Cumba, } \\
\text { Guavito, Quebrada Los Chorros, El } \\
\text { Alto, La Isabela, Fondo Ganadero, } \\
\text { La India, Hacienda el Medio, Colora- } \\
\text { da, Zambrano, El Garcero, La Flores- } \\
\text { ta, Valparaíso y La Esmeralda }\end{array}$ & $\begin{array}{l}03^{\circ} 12^{\prime} 02^{\prime \prime} \text { Latitud Norte y } 76^{\circ} 28^{\prime} 18^{\prime \prime} \\
\text { Longitud Oeste }\end{array}$ \\
\hline Bolo Artonal & Pradera & Corregimiento de Bolo Artonal & $3^{\circ} 26.8424^{\prime}$ Latitud Norte, $76^{\circ}$ \\
\hline Cañas Abajo & Florida & $\begin{array}{l}\text { Corregimiento de Cañas Abajo, vere- } \\
\text { da perteneciente al corregimiento de } \\
\text { El Remolino }\end{array}$ & $\begin{array}{l}16.0211^{\prime} \text { Longitud Oeste } \\
\text { Latitud de } 3^{\circ} 19^{\prime} 0.01^{\prime} \text { una Longitud } \\
\text { de }-76^{\circ} 16^{\prime} 0.01\end{array}$ \\
\hline Tarragona Alta & Florida & Corregimiento de Tarragona Alta & $\begin{array}{l}\text { Latitud de } 3.28333 \text { y Longitud de } \\
76.35\end{array}$ \\
\hline
\end{tabular}

las comunidades negras: interculturalidad, suficiencia de información, consentimiento previo, no imposición de criterios unilaterales, no a la intermediación económica, bienestar colectivo, respeto por la diferencia, co-responsabilidad, generación y cumplimiento de acuerdos, perspectiva de consensos.

A continuación, se definen cada uno de estos principios, desde la percepción y conceptualización de los participantes en los diferentes talleres.

Principio de interculturalidad. Se basa en el reconocimiento, respecto y valoración de la presencia e interacción de las diversas culturas, dentro de su cosmovisión, así como la posibilidad de generar expresiones culturales compartidas, adquiridas por medio del diálogo y de una actitud de respeto mutuo. Se reconocen los conocimientos tradicionales en el manejo y uso de los recursos forestales y de fauna silvestre y de la biodiversidad. Además se busca promover el fortalecimiento de aptitudes y competencias recíprocas para una comprensión intercultural entre el Estado, los pueblos indígenas y la sociedad en su conjunto.

Durante la ejecución de cualquier proyecto o actividad que involucre relacionamiento con comunidades negras, es importante aplicar este principio que permitirá una óptima interacción con los miembros de las comunidades negras, requisito indispensable para poder solidificar una relación armoniosa de trabajo y poder concretar los objetivos y obtener los resultados esperados por el equipo técnico del proyecto o actividad.

Información suficiente y oportuna. Este principio Busca garantizar que la información 
concerniente al proyecto o actividades a desarrollar dentro de los territorios comunitarios sea suministrada de manera clara y oportuna, utilizando un lenguaje adecuado a las características del contexto comunitario en el cual se desarrolla, además de garantizar que la información esté disponible a todas las instituciones participantes y actores comunitarios a fin de que pueda ser analizada de manera diáfana y poder manifestar sus puntos de vista.

Consentimiento previo. Con este principio se busca que las comunidades tengan el derecho a determinar si y bajo qué condiciones se deben llevar a cabo procesos de desarrollo locales que puedan afectarlos. Se dará especial énfasis a establecer un consentimiento previo al inicio de un proyecto, pero el proceso de consentimiento no termina al conseguir la aprobación de la comunidad para seguir adelante con el inicio de un proyecto. Un componente esencial de este proceso en marcha es el establecimiento de mecanismos de monitoreo participativos e independientes que monitorearán el acatamiento por la parte de los actores interesados de los acuerdos y compromisos con la comunidad (y viceversa) y servirá como un medio para resolver quejas o disputas que puedan surgir.

No imposición de criterios unilaterales. Referido a la forma de reunir en el diálogo a todos aquellos que puedan ser parte de un proceso de relacionamiento entre organismos estatales y grupos comunitarios, mediante la negociación de significados y formas de significar, es decir, sin que medie la imposición de criterios por ninguna de las partes, permitiendo abrir la puerta a una participación equitativa y efectiva de todos los actores.

No intermediación económica. La cosmovisión de las comunidades afrocolombianas del Pacífico, representa rupturas sustanciales en los conceptos de dinero. En consecuencia, representa una ruptura con el pensamiento occidental y con el modelo social que este impulsa. El valor de acumulación es algo externo a la dinámica de la mayoría de las comunidades afrocolombianas. Para la comunidad afro, en su cotidianidad, el dinero tiene su mayor valor en la facilitación del intercambio entre productos y actividades. El principal valor de acumulación sigue siendo representado por la abundancia de recursos y servicios ambientales. Bajo estas concepciones no se admite ningún tipo de relacionamiento y/o negociación con comunidades negras que anteponga la intermediación económica para la consecución de acuerdos

Bienestar colectivo. La construcción de bienestar colectivo es uno de los propósitos fundamentales del actuar político y organizativo de las los Consejos Comunitarios de Comunidades Negras, fundamentado en la capacidad cultural y ambiental de sus territorios ancestrales para garantizar la satisfacción material y espiritual, en especial a los habitantes de las comunidades negras del Valle del Cauca, sin importar sexo ni edad. Es en los postulados del bienestar colectivo, se inspira la capacidad de gestión y evolución como grupo étnico.

Respeto por la diferencia. Este principio se establece como la dirección que facilitará la planeación, atención de necesidades y apropiación de los recursos necesarios para el establecimiento del ejercicio de negociación y acuerdos colectivos a partir de sus características y necesidades propias con criterios de igualdad de condiciones y oportunidades, equidad y justicia.

Co-responsabilidad, generación y cumplimiento de acuerdos. Las comunidades negras se caracterizan por tener un elevado sentido del cumplimiento de la palabra en los compromisos asumidos, tanto a nivel personal como colectivo. En tal sentido este principio constituye un pilar fundamental para llevar a cabo cualquier tipo de relacionamiento con consejos comunitarios, en tanto que no sólo garantiza el establecimiento de sólidas relaciones de confianza y credibilidad entre las organizaciones comunitarias y los 


\section{Bioetnia Volumen 12, 2015}

demás involucrados, sino también la co-responsabilidad en el cumplimiento de los acuerdos, actividades y tareas comprometidas en el marco del relacionamiento y en coordinación con la entidad ejecutora y las instituciones financieras. Además, estos compromisos y responsabilidades serán adecuadamente formalizados mediante la generación de acuerdos refrendados con actas y firmas de los participantes.

Perspectiva de consensos. Bajo este principio se plantea una metodología de trabajo colectivo y no de forma individual, porque es a partir del trabajo colectivo que se expone desde distintos puntos de vista, una realidad compartida y se van construyendo diagnósticos y soluciones conjuntas. Es decir, consulta directamente a las personas involucradas en el desarrollo de un proceso y lo hace para garantizar que sus opiniones y aportes sean escuchados, discutidos y tenidos en cuenta, a la hora de formular propuestas para afrontar dificultades, buscar soluciones a sus propios problemas o necesidades. También para propiciar la concertación entre diversos intereses, con el fin de conseguir objetivos comunes.

\section{Elementos relevantes de la cultura afrodescendiente a ser considerados}

Los participantes de los talleres, de manera general y en consenso, coinciden en el hecho que para iniciar una adecuada y armoniosa relación con los habitantes de las comunidades negras, es necesario comprender los elementos que los distinguen de manera particular, considerando sus propias percepciones y conceptos acerca del mundo, de la naturaleza y formas de vida que constituyen su cultura. Por ello, plantean como elementos relevantes que deben ser considerados de la cultura afro en relación con la gestión de los recursos naturales, los siguientes:

- Relación integral del hombre con la naturaleza: se reconoce que la naturaleza es un ser vivo y el hombre forma parte de ese sistema de vida.

- Los territorios colectivos son de dominio y propiedad colectiva, pertenecen a todos los miembros del consejo comunitario y su uso es definido colectivamente mediante acuerdos de asamblea general o según lo estipulado en el reglamento interno de cada consejo comunitario, en concordancia con las prácticas tradicionales y costumbres.

- La clasificación de las tierras se da según los usos definidos de manera ancestral y tradicional, como por ejemplo las áreas protegidas y de interés ambiental, de uso sostenible, entre otras, que se respetan por las generaciones presentes y futuras.

- El uso de los recursos naturales se realiza de manera racional y equitativa por todos los miembros del consejo comunitario conforme a las necesidades de todos los miembros de la comunidad. Por ello, se racionaliza (mide) los recursos a fin de asegurar la satisfacción de necesidades presentes y futuras de todos los miembros de la comunidad se cuente o no con territorio colectivo.

- Considerar que el reglamento interno de cada consejo comunitario establece la organización social, económica, política y cultural dentro de la comunidad y garantiza la gobernanza comunitaria.

\section{Aspectos de sensibilidad cultural a tener presente}

Los pueblos afrodescedientes tienen un arraigo espiritual con sus territorios, en los que pueden desarrollar sus prácticas tradicionales y costumbres y mantienen su cultura; es por ello que los miembros de los consejos comunitarios coinciden en que en necesario tener presente los siguientes aspectos de sensibilidad cultural:

- Reconocer que se está frente a una cultura diferente a la propia. 
- Observar con detenimiento a fin de apreciar (sin hacer juicios valorativos) e intentar comprender y aprender las formas, gestos, actitudes y expresiones de comunicación de la población afro.

- Reconocer y respetar las actividades realizadas por la población afro, así como los tiempos que le dedican a dichas actividades.

- Comprender que las comunidades negras han aceptado vivir con un vínculo con los bosques, en tanto constituyen su hábitat, aun cuando no cuenten con condiciones básicas de saneamiento, es el ambiente en la que viven, y es necesario respetar esa forma de vida.

- Interpretar las necesidades o limitaciones del miembro del consejo comunitario: materiales (carencia de servicios básicos y servicios de salud) y educativas y formativas.

\section{Conductas sugeridas para la interacción con comunidades negras}

- Al momento del diálogo personal, comunicación social, extensión o divulgación de contenidos temáticos, usar lenguaje sencillo y de ser posible con ejemplos o casuística.

- Fortalecer la confianza con los pobladores afro mediante una actitud y capacidad de escucha activa con el interlocutor afrodescendiente.

- Respetar los usos y costumbres de la comunidad dependiendo de las circunstancias y en lo posible tratar de acompañar vivencialmente, por ejemplo, práctica de danzas, preparación de alimentos, etc.

- El equipo técnico del proyecto no debe mostrar actitudes de menosprecio o de rechazo, ni tampoco signos de superioridad en razón de su grado de formación profesional frente a los miembros de comunidades negras. Se insta a los miembros del equipo técnico a incentivar a que los llamen o identifiquen por su nombre más que por el título o grado (abogado, ingeniero, etc.). Evitar actitudes de soberbia o discriminación que puedan generar confrontaciones.

- Hacer el esfuerzo por adaptarse a las condiciones de la comunidad: servicios públicos deficientes, no hay policía, no hay servicios de energía, telefonía, agua y saneamiento, no hay viviendas particulares, entre otras.

- Hacer el esfuerzo por adaptarse a las condiciones climáticas y del ambiente (condiciones agrestes, lluvias, alta temperatura, radiación, incidencia de riesgos a la salud, etc.).

- Generar condiciones de participación activa de las mujeres, jóvenes, ancianos, en los procesos de reflexión y formulación de las propuestas de solución de su problemática y realidad del territorio.

\section{Pautas claves de relacionamiento}

En procura de una eficiente relación con comunidades negras y en consonancia con los principios de participación, transparencia, equidad, visión estratégica, co-responsabilidad y cumplimiento de acuerdos, vocación de servicio y reciprocidad e interculturalidad, se puede agrupar en cuatro fases o componentes metodológicos (Figura 1), cada uno de los cuales fueron analizadas por separado:

- Identificación de actores claves en los consejos comunitarios

- Divulgación de información

- Concertación y socialización con actores claves y demás actores comunitarios

- Suscripción de acuerdos y compromisos

Identificación de actores claves en los consejos comunitarios. El primer paso del proceso para establecer relaciones con los consejos comunitarios es la participación comunitaria; en este sentido es importante iniciar por la identificación 


\section{Bioetnia Volumen 12, 2015}

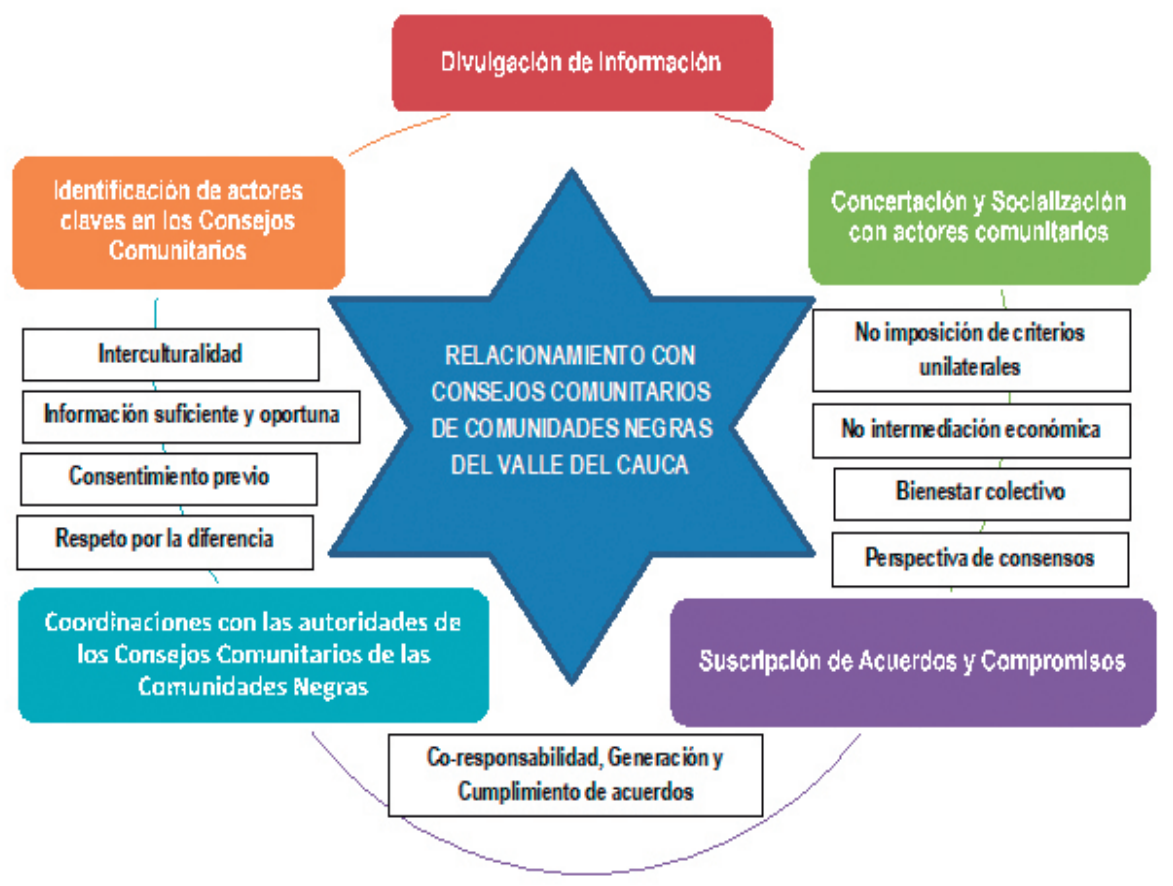

Figura 1. Componentes metodológicos para el relacionamiento con comunidades negras.

de actores claves, es decir, los miembros de las juntas directivas de los consejos comunitarios, es con ellos con quienes se debe realizar las consultas iniciales, porque ellos constituyen un medio eficiente para difundir información al resto de los miembros del consejo comunitario. Sin embargo, hay varios factores que se deben tener en cuenta a la hora de interactuar con los actores claves, es importante conocer que ellos como representantes legales de los consejos comunitarios, representan los intereses y están facultados por la normatividad vigente en esta materia, además de ser ellos verdaderos defensores de las opiniones de sus representados, y que se pueda confiar en que transmitirán fielmente a sus representados los resultados del diálogo con las partes interesadas para el desarrollo de los proyectos o actividades en sus territorios colectivos.

Se recomienda interactuar con los actores sociales en sus propias comunidades, pues es allí donde ellos se sienten más cómodos y con toda seguridad, un lugar en su propia comunidad, tienden a entablar relaciones más productivas, por las siguientes razones:
- Da transparencia al proceso. Los miembros de la comunidad pueden ser testigos del proceso y mantenerse informados sobre las conversaciones que se llevan a cabo en su representación y sobre los acuerdos alcanzados al término de las consultas o negociaciones.

- Da a entender que los actores interesados valoran la opinión de las comunidades al punto de estar dispuestas a viajar al lugar y pasar allí el tiempo necesario.

- Contribuye a que los miembros de la comunidad se sientan identificados con el proceso. Los miembros de la comunidad señalan que cuando se les da la oportunidad de expresar sus puntos de vista en las reuniones públicas, sienten que su participación es importante para el resultado de las decisiones adoptadas.

Coordinaciones con las autoridades de los consejos comunitarios de las comunidades negras. Toda vez que sea necesario tratar algún tema con las comunidades $\mathrm{u}$ organizaciones afrodescendiente, se remitirá una carta formal del 
proyecto a las autoridades que integran la junta directiva, a fin de presentar de manera genérica el proyecto y proponer que se conceda una fecha para sostener reuniones de coordinación en función con la agenda propuesta. Recibida la carta de respuesta de las autoridades de la comunidad $u$ organización afrosdencendiente, la coordinación del proyecto determina quienes participarán de esa reunión. La coordinación del proyecto informa de las coordinaciones de todo el equipo técnico a fin de que puedan dar sus aportes.

Divulgación de información. Divulgación de información es una expresión formal que significa dar acceso a información a los actores interesados y afectados por un proyecto. La comunicación de esa información de una manera comprensible para los actores comunitarios es un primer paso importante (y continuo) en las relaciones con los actores comunitarios. Todas las demás actividades, desde las socializaciones y la participación con conocimiento de causa hasta la concertación, serán más constructivas si los actores comunitarios, cuentan con información precisa y oportuna sobre el proyecto, su impacto y otros aspectos que pudieran producir algún efecto en ellos.

En consecuencia y como estrategia recomendada, se propone adoptar medidas para aumentar la transparencia y la entrega de información significativa y confiable como medios de promover un entendimiento acerca del proyecto e infundir confianza a los actores comunitarios. Adoptar una presunción a favor de la divulgación significa dar a conocer información cada vez que sea posible, sobre todo si no hay razones de peso para no difundirla.

Divulgar información implica presentación del proyecto o actividades a llevar a cabo en los territorios, dependiendo de las facilidades logísticas de la zona; la presentación del proyecto se puede hacer utilizando diversos recursos:

- Una presentación en PowerPoint, versión de formato amigable y conciso

- Una presentación en Word, versión de formato extendida

- Papelógrafos

La presentación del proyecto se debe enfocar en poder expresar de manera clara y sencilla los objetivos, principios, componentes y resultados esperados en el plazo de implementación del mismo, considerando que si bien un proyecto no puede solucionar toda la problemática de las comunidades, si puede contribuir a mejorar las condiciones que permitan a los miembros de la comunidad a adquirir nuevas capacidades, contar con mejores oportunidades de empoderarse y responsabilizarse de su propio desarrollo.

De otro lado, los proyectos o propuestas de actividades, son siempre contingentes, quedando abierta la posibilidad de revisar sus supuestos y componentes, de retroalimentar la información y de hacer ajustes intermedios para mejorar las estrategias. Para ello el proyecto cuenta con mecanismos de monitoreo y gestión adaptativa permanente.

Socializaciones y concertaciones con actores claves y demás actores comunitarios. En este sentido, se propone realizar las socializaciones y concertaciones aplicando los principios básicos de las prácticas recomendadas por el IIAP a partir de su experiencia en relacionamiento con actores comunitarios de los valles interandinos, desde donde se ha definido que no existe una sola forma correcta de realizar las socializaciones y concertaciones. Por su naturaleza, el proceso siempre dependerá del contexto. Esto significa que las técnicas, métodos, estrategias y calendarios deberán adaptarse a la situación local y a los diversos actores comunitarios con quienes se han de realizar las socializaciones y concertaciones. Idealmente un proceso de socialización y concertación bien concebido:

- Se lleva a cabo con la debida anticipación para tener en cuenta las cuestiones fundamentales y para que repercuta en las decisiones relativas al proyecto o actividades en procesos de socialización y concertación. 


\section{Bioetnia Volumen 12, 2015}

- Es un proceso libre e informado, como resultado de la divulgación por anticipado de la información pertinente.

- Es significativo para las comunidades con quien se socializa y concerta porque su contenido se presenta en un formato fácil de comprender y las técnicas empleadas son culturalmente apropiadas y contextualizadas.

- Es bidireccional, de manera que ambas partes tienen la oportunidad de intercambiar opiniones e información, escuchar y plantear sus inquietudes para que sean resueltas;

- Es inclusivo desde el punto de vista del género, al tener en cuenta que a menudo los hombres y las mujeres tienen distintas perspectivas y necesidades.

- Se adapta a las condiciones del lugar (costumbres locales, calendarios y contexto adecuados).

- Queda documentado especialmente en actas para llevar cuenta de los resultados de socialización y concertación.

- Es un proceso continuo durante la vida del proyecto, según las necesidades.

Suscripción de acuerdos y compromisos. Los resultados de la socialización y concertación planteados, se evidencian en los acuerdos y compromisos a que se llega finalmente con los actores comunitarios; en este aspecto se plantea como principio fundamental la realización y suscripción de acuerdos y compromisos con los actores comunitarios "de buena fe", es decir, con amplitud de criterio, voluntad de participar en el proceso y el deseo genuino de llegar a consensos que se traduzcan en acuerdos y compromisos. La suscripción de acuerdos y compromisos realizadas de buena fe son transparentes, toman en cuenta el tiempo de que disponen los involucrados y hacen uso de procedimientos y de un lenguaje fácilmente comprensible para todas las partes y acordados entre ellas. Los principios clave que se deben tener presentes son:

- Participación de representantes legítimos

- Uso de estrategias participativas

- Disponibilidad de tiempo suficiente para la toma de decisiones

- Respeto mutuo y consideración de las diferencias culturales y de otra índole

- Flexibilidad, análisis de diversas opciones y voluntad de alcanzar acuerdos y compromisos

- Las presentaciones del proyecto deben desarrollarse de manera dialogada y pausada.

- Los argumentos, ideas y comentarios deben ser transmitidos de la manera más sencilla posible, evitando el uso de tecnicismos o abstracciones.

- Parailustrar las presentaciones, preferentemente usar medios gráficos e interactivos (papelógrafos, cartulinas de colores, proyecciones en PowerPoint, videos, otros).

- Con el fin de promover la inclusión de las comunidades negras en el proyecto se presentan ejemplos motivadores de otras comunidades donde se hayan implementado de manera efectiva y sostenible experiencias de manejo ambiental del territorio y procesos etnoeducativos y de fortalecimiento de la gobernanza. Asimismo, fundamentar la importancia del componente de formación de líderes como un valor esencial para el fortalecimiento de las organizaciones afrodescendientes.

- Promover el diálogo fluido, de preferencia en su idioma, como primera instancia y luego propiciar las reflexiones sobre los temas de interés teniendo en cuenta el relacionamiento intercultural. Entrega de materiales impresos.

- Con el fin de lograr un acuerdo con los miembros de las comunidades negras, se debe dar a conocer, de manera transparente y sincera, clara y precisa toda la información que está inmersa en el mensaje, 
para que comprendan todo lo que abarca el acuerdo y pueda trabajarse de manera conjunta.

- Finalmente se recomienda la formalización de los acuerdos y compromisos con las comunidades negras mediante la suscripción de actas debidamente firmadas por cada una de las partes involucradas.

Construcción de la estrategia para resolver las situaciones ambientales a través de procesos etnoeducativos con comunidades negras de consejos comunitarios

Una vez definidos los principios y pautas claves de relacionamiento, al igual que los mecanismos de negociación y suscripción de acuerdos con los consejos comunitarios, se propuso la siguiente ruta estratégica para resolver las situaciones ambientales a través de procesos etnoeducativos con estas comunidades.

La implementación de estrategias educativas dirigidas a la solución de situaciones ambientales con comunidades negras, implica que se asuman procesos de larga duración y un trabajo sobre distintos ejes a la vez. La experiencia muestra que los procesos rápidos fracasan y se requieren procesos lentos y de larga duración; uno de los aspectos que deben resultar positivos es la relación entre costos y beneficios de las estrategias que se busquen implementar, lo cual exige construir soluciones nada sencillas en contextos complejos. En todos los casos es importante considerar las particularidades del contexto.

En tal sentido se proponen dos grandes estrategias: El desarrollo permanente de capacidades en las comunidades y la construcción de escenarios de diálogo o mesa ambiental comunitaria.

Desarrollo permanente de capacidades en las comunidades. Los retos que implica generar procesos etnoeducativos para resolver situaciones ambientales en el contexto de comunidades negras, requieren de habilidades que las comunidades en este momento solo tienen parcialmente. Es importante que se desarrolle una estrategia educativa y de formación. En primer lugar, muchas de las acciones serían muy útiles si se desarrollan con las escuelas, aprovechando que los profesores pueden jugar un papel importante, no solo con los niños sino también con los padres de familia.

El amplio conocimiento en las comunidades negras asociado con el manejo de los recursos naturales no ha sido suficiente para conservar los modelos tradicionales de aprovechamiento de los recursos naturales que se han sido objeto de transformación debido a presiones externas de orden económico, social y cultural, aumentando la amenaza sobre estos recursos. Es, por lo tanto, necesario contar con herramientas participativas para la recolección de información, que conjuguen el conocimiento tradicional con los elementos técnicos y que a su vez permitan el análisis y toma de decisiones concertadas para una eficiente gestión y conservación del medio ambiente.

Son muchos los conceptos que las comunidades deben manejar y conocer sus características como todo lo relacionado con los reconocimientos territoriales, los ejercicios de planeación propias y de otras entidades territoriales, asuntos relacionados con el ordenamiento territorial y ambiental y la legislación ambiental. También debe entender conceptos como los servicios ambientales. Por otro lado, requieren comenzar a manejar formas de manejo forestal que pueden incluir el manejo de viveros, ejercicios de reforestación y protección y recuperación de fuentes hídricas, cambio climático, entre otros.

La construcción de escenarios de diálogo o mesa ambiental comunitaria. El desarrollo comunitario incorpora acciones y decisiones de actores que buscan incidir positivamente en el entorno y lograr transformaciones dirigidas a mejorar la calidad de vida de los habitantes. Desde esta perspectiva, el conocimiento del contexto social, cultural y natural, además de las múltiples potencialidades del territorio, se convierten en el punto de partida para generar propuestas de participación ciudadana, donde las mesas ambientales 


\section{Bioetnia Volumen 12, 2015}

comunitarias juegan un papel determinante, pues se han venido consolidando como espacios de encuentro ciudadano para la reflexión de situaciones ambientales presentes en el territorio y para la gestión ambiental participativa.

La mesa ambiental comunitaria puede ser el espacio en el cual las comunidades negras y los sujetos del territorio tienen la oportunidad de capacitarse, desarrollar procesos comunicativos, implementar acciones de prevención, incidir en la planificación, formular proyectos, además de mitigar problemas. Para llevar a cabo estas estrategias se parte de las reflexiones generadas en las preocupaciones por las condiciones del contexto; a la vez se puede utilizar, una herramienta de la educación ambiental que es la lectura del contexto, con la cual los sujetos adquieren un conocimiento mínimo de lo que sucede.

Poner en funcionamiento una mesa ambiental comunitaria implica la identificación de los actores claves: organizaciones territoriales como los consejos comunitarios, estatales como la CVC, IIAP (2015), alcaldías municipales; gremiales como las organizaciones de campesinos, ganaderos, azucareros, actores asociados con la educación con intereses en el tema ambiental, así como ONG interesadas en el tema ambiental del Valle del Cauca.

Por un lado, esta mesa puede ser un buen espacio para que los consejos comunitarios presenten y compartan sus inquietudes y perspectivas, además de que logre generar una visión integral sobre el territorio con la participación de todos.

En estas mesas se pueden construir mediante ejercicios participativos como diagnósticos del estado de los recursos y la construcción colectiva de cartografías sociales. Entender las bases de los problemas ambientales les permite a los sujetos del territorio tener un mayor criterio para desarrollar alternativas para la solución, a la vez para pretender incidir en las decisiones ambientales que se requieren para mejorar los niveles de relacionamiento con los ecosistemas existentes en el territorio. En este caso se pueden desarrollar ejercicios como el análisis de las debilidades, oportunidades, fortalezas y amenazas sobre el territorio y sus diferentes recursos. También es posible construir diagramas de causa-efecto, llamado diagrama espina de pescado; es una forma de organizar y representar las diferentes causas de un problema y evaluar si su solución está al alcance del grupo.

Este diagrama ayuda a poner de relieve y analizar las causas del problema. Se denomina «espina de pescado» por la forma en que se van ubicando cada una de las causas o razones que originan un problema. Permite visualizar de una manera rápida y clara la relación que tiene cada una de las causas con las demás razones que inciden en el origen del problema. En algunos casos, son causas independientes y, en otros, existe una íntima relación entre ellas, que pueden estar actuando en cadena.

La gráfica, resultado de este método, tiene un eje central horizontal, conocido como «espina central», y varias flechas inclinadas que se extienden hasta el eje central, en dirección de la «boca del pez», donde se encuentra el problema analizado; cada espina contiene y representa un grupo de causas que inciden en la existencia del problema.

Esta herramienta permite identificar, explorar y representar en grupo y de manera rápida todas las posibles causas de un problema con el fin de descubrir sus raíces. Por otra parte, permite concentrarse en el contenido del problema, no en la historia o intereses personales asociados con él. Además, al ser una «fotografía» del problema elaborada con la participación de todos, se convierte en un apoyo para buscar soluciones de manera consensuada.

Para poder elaborar este diagrama, es necesario definir primero el problema que va a ser analizado. Identificado el problema, se dibuja en el tablero o papel el esqueleto de la espina de pescado y ubicamos en la cabeza del pescado el problema 
identificado, de la manera más sencilla y breve posible. Luego, con base en las observaciones, conversaciones y datos recogidos, se trata entre todos de identificar el mayor número de causas que están contribuyendo a generar este problema. La pregunta de «por qué está sucediendo...» puede ser de gran ayuda. Entonces se agrupan las causas en categorías. Para comprender mejor el problema, se buscan las subcausas o razones de esas causas principales. Si es necesario, se puede elaborar un diagrama causa-efecto para cada una de las causas. Recuerde que se trata de obtener una comprensión más clara y precisa; sin embargo, use su sentido común para detenerse.

Cuando tenemos un panorama de las causas, las ordenamos en grupos de acuerdo con categorías para facilitar la búsqueda de soluciones; por ejemplo: las causas que se pueden resolver de manera comunitaria o con ayuda externa. El propósito es definir si la solución de este problema depende del grupo o no está a su alcance.

El diagrama causa-efecto o espina de pescado busca las raíces del problema, va de las causas principales a causas más profundas para facilitar la identificación de soluciones, que, como en este caso, pueden conducir a un plan de trabajo para constituir un proyecto de formación o producción en el que se compartan saberes y prácticas con las comunidades.

\section{Conclusiones}

El enfoque de acercamiento a las comunidades negras que aquí se plantea no es únicamente de naturaleza epistemológica, sino primordialmente existencial. La presente estrategia metodológica tiene como finalidad promover una comunicación empática, asertiva, fluida, sencilla y comprensible por parte de los diferentes entes gubernamentales y demás organizaciones hacia los integrantes de las comunidades, de manera que se logren condiciones favorables de confianza mutua que favorezcan el trabajo conjunto.
En consecuencia, esta estrategia metodológica tiene por objeto ser una herramienta práctica y de uso sencillo para ayudar a la organizaciones públicas o privadas a entender la necesidad de conocer y respetar las complejidades culturales, sociales, económicas y políticas relacionadas con la realización de proyectos y demás actividades en territorios colectivos de comunidades negras. El propósito es que esta estrategia plantee enfoques positivos, prácticos y generales que permitan a las organizaciones públicas o privadas establecer relaciones fructíferas con los consejos comunitarios de comunidades.

Sin embargo, en vista de su rica diversidad, no puede haber un enfoque único cuando se establecen relaciones con los consejos comunitarios. Esta estrategia no trata sólo del establecimiento de buenas relaciones entre una organización pública o privada y un consejo comunitario, sino que es un medio para ayudar a las organizaciones a iniciar o mejorar el proceso de construir una relación de manera respetuosa y positiva.

Es importante que en la implementación de estos procesos etnoeducativos para la resolución de situaciones ambientales en los Consejos Comunitarios de los Valles Interandinos-Cuenca Cauca, tenga presente algunos elementos para favorecer el éxito de las acciones o estrategias.

En lo institucional. Será necesario buscar la consolidación del ejercicio de derechos territoriales, no solo de las comunidades negras sino de otros actores que dependen directamente de su subsistencia de este territorio como los colonos, ingenios azucareros, ganaderos, entre otros. En este esfuerzo es importante contribuir a que se tenga acceso a derechos formales de uso de recursos forestales y por lo tanto que se logre involucrar la institucionalidad competente.

En lo económico. Es importante que se ayude a la generación de fuentes propias de trabajo e ingresos y que las acciones que se desarrollen ayuden al mejoramiento de los medios de vida de la población local. 


\section{Bioetnia Volumen 12, 2015}

En lo social. Para aportar al bienestar social es importante contribuir con el fortalecimiento de la organización de la comunidad y en este sentido ayudar a fortalecer las capacidades de autogestión. También será importante buscar la equidad en la distribución de beneficios derivados de una buena gestión ambiental del territorio.

En lo ambiental. En todo momento es importante reflexionar y buscar que se aporte a la conservación de la biodiversidad y tal vez más importante para la misma población, lograr el mantenimiento de los servicios ambientales, y con esto contribuir en una buena gestión ambiental del territorio a la solución de sus problemáticas ambientales

\section{Literatura citada}

Corporación Autónoma Regional del Valle del Cauca, Instituto de Investigaciones Ambientales del Pacífico. 2015. Implementación de procesos etnoeducativos ambientales con comunidades negras del Valle del Cauca, orientado hacia la recuperación de áreas de interés ambiental, recuperación de microcuencas abastecedoras de acueductos, manejo adecuado de residuos sólidos a través de procesos participativos para la gestión ambiental. Informe final, Convenio 033 de 2013. Chocó: CVC-IIAP.

Corte Constitucional de Colombia. Constitución Política de Colombia. URL disponible en: http://www.corteconstitucional.gov.co/inicio/Constitucion $\% 20$ politica $\% 20$ de $\% 20$ Colombia $\% 20-\% 202015$.pdf

SERVINDI. 2005. Interculturalidad: Desafio y proceso en construcción. Manual de capacitación. Lima-SERVINDI; 122.

UNESCO, Organización de las Naciones Unidas para la Educación, la Ciencia y la Cultura. 2009. Informe mundial de la UNESCO. Invertir en la diversidad cultural y el diálogo intercultural. Resumen. París: UNESCO. URL disponible en: http://unesdoc.unesco. org/images $/ 0018 / 001847 / 184755 \mathrm{~s}$.pdf

UNESCO. 2001. Declaración Universal de la UNESCO sobre la Diversidad Cultural. Conferencia General de la UNESCO en su $31^{\text {a }}$ reunión celebrada el 2 de noviembre de 2001 en París. URL disponible en: http://portal. unesco.org/es/ev.php-URL_ID $=13179 \& U R L \_D O=-$ DO TOPIC\&URL_SECTION=201.html

UNESCO. 2005. Convención sobre la Protección y Promoción de la Diversidad de las Expresiones Culturales. Conferencia General de la UNESCO, en su $33^{\text {a }}$ reunión celebrada entre el 3 y 21 de octubre de 2005 en París; 19 pp. URL disponible en: http://unesdoc.unesco.org/ images/0014/001429/142919s.pdf 